RESEARCH ARTICLE

\title{
Mysticism and the Fine Structure Constant
}

\author{
PhiLIP R. BROWN \\ Department of Foundational Sciences, Texas A\&M University at Galveston \\ brownp@tamug.edu \\ Submitted January 31, 2018; Accepted March 5, 2020; Published September 15, 2020 \\ https://doi.org/10.31275/20201289 \\ Creative Commons License CC-BY-NC
}

\begin{abstract}
The mysticism relating to the fine structure constant, including the surprising connection between the number 137 and Kabbalah by means of gematria (first noted by Gershom Scholem in the 1950s), and the connection between Wolfgang Pauli's World Clock dream and formulas for the fine structure constant, is explored and expanded. A formula that relates the number 137 to the golden ratio and the Fibonacci sequence is explored in the context of Platonic and Pythagorean mysticism and various religious and esoteric traditions.

Keywords: fine structure constant; pi; quantization; archetypes; Kabbalah; polygons; Pythagoras; Plato; Carl Jung; mysticism; golden ratio; Fibonacci numbers; gematria
\end{abstract}

\section{INTRODUCTION}

An historical and ongoing aspiration of physicists is to express certain fundamental constants ${ }^{1}$ or ratios (for example, the coupling constants and the ratio of the proton mass to the electron mass) in terms of simple formulas involving integers or transcendentals like $\pi$ and $e$.

For example, in 1913 Gilbert N. Lewis and Elliot Q. Adams developed their theory of ultimate rational units, a system in which all universal constants could be expressed in terms of only integral numbers and $\pi$ (Lewis \& Adams, 1914). Before and since there have been many other attempts. Some references are Dattoli (2010), Krag (2003), Lunn (1922), Sherbon (2008, 2014), Stanbury (1983), and Várlaki et al. (2009b). 
Philip R. Brown

\section{The Fine Structure Constant}

A particular constant that has been the focus of these aspirations is the fine structure constant, usually denoted $\alpha$, and defined by the formula

$$
\alpha=\frac{e^{2}}{n c},
$$

where $e$ is the charge of an electron, $h$ is the reduced Planck's constant, and $c$ is the velocity of light in a vacuum. ${ }^{2}$ It has been known since the 1920 s that $\alpha$ has the approximate value $1 / 137$.

The values for $\alpha$ and its reciprocal, based on experimental measurement using the quantum Hall effect or the anomalous magnetic moment of the electron (see Kinoshita, 1996, 1997), that are recommended by the Committee on Data for Science and Technology (CODATA), an interdisciplinary committee of the International Council for Science, in 2014, are

$$
\alpha=0.0072973525664(17)
$$

and

$$
\alpha^{-1}=137.035999139(31)
$$

(the numbers in parentheses specify the uncertainty in the last two digits). The above value for $\alpha^{-1}$ has recently been closely matched by employing measurements of cyclotron frequencies of single ions in Penning traps to obtain precise atomic mass measurements (Myers, 2019).

On the other hand, using the current recommended CODATA values (using cgs units) $e=4.80320425(10) \times 10^{-10}$ statC, $h=$ $1.054571800(13) \times 10^{-34}$ joule seconds, and $c=29979245800 \mathrm{~cm} / \mathrm{s}$, the fine structure constant and its reciprocal have the following values with the indicated uncertainty:

$$
\alpha=0.00729735127(40)
$$

and

$$
\alpha^{-1}=137.036023(07)
$$


Recent astronomical observations of the spectra of distant quasars suggest that $\alpha$ has a spatial or temporal variation of at most .001\% since the beginning of the universe (about 13.7 billion years ago) (Cowie \& Songaila, 1995; Savedoff, 1956; Webb et al., 1998, 2001, 2012). From the analysis of shifts of thermal neutron-capture resonances of rare earth elements of natural fission reactors which operated about 2 billion years ago at Oklo (in Gabon), it has been determined that the time variation of $\alpha$ since then is about $0.00001 \%$ (Damour \& Dyson, 1996).

There have also very recently been reports of evidence for a varying speed of light, for example by Afshordi and Magueijo (2016) who suggested that the speed of light was higher when the universe was in its infancy. In some other theories, the speed of light could be decreasing gradually (Barrow, 1999).

As evidenced by the extensive literature on the topic, there have been many interesting attempts to produce a simple and correct expression for $\alpha$ on the basis of a theory of physics and in accordance with historical, aesthetic, and philosophical expectations. Indeed, as we will elucidate below, there has developed a mythos relating to $\alpha$ which seems to have very ancient roots in mysticism and alchemy and, in particular, to the connection of the number 137 to Kabbalah. The number 137 has also permeated popular culture. ${ }^{3}$

This article should be read in the context of a non-materialistic or non-naturalistic view of Nature. This would include Carl Jung's idea of the collective unconscious that is responsible for universal cultural images or symbols that he referred to as archetypes. Furthermore, the formulas and numerical "coincidences" that we discover in this article are inspired by and interpreted within a cultural and historical context; for example, formula (7) below is derived from a value for the fine structure constant suggested by the physicists Lunn and Heisenberg (as explained in the next section) and interpreted in terms of Wolfgang Pauli's World Clock Dream; and the formula $137=5+24+108$ which relates the fine structure constant to the Fibonacci sequence in an intriguing way is interpreted in terms of Pythagorean mysticism, Plato's mystical numbers, special numbers in religion, and gematria. Why these numerical "coincidences" relating the fine structure constant, classical culture, and (esoteric) religion should exist, we do not speculate about besides mentioning Jung's proposal of the unus 
mundus, of which the small counting numbers are the archetypes, and the related Pythagorean belief that numbers mediate between the divine and physical realms.

While mystical tradition and science apparently seek truth within different belief systems and epistemologies, there is some evidence (Werner's Heisenberg's experience in India that we will mention below, for example) for a commonality to be found between them. Numerology could be one place to look for it.

\section{The Lunn-Heisenberg Constant}

In this article we investigate the number

$$
\frac{\pi / 2}{6^{3}}=0.007272205621 \ldots
$$

which was introduced in 1922 as one of a few possible algebraic expressions for $\alpha$ by the Chicago physicist Arthur C. Lunn (Lunn, 1922). It was also suggested independently by Werner Heisenberg in 1935 when he stated the following in a letter to Neils Bohr (Heisenberg, 1934):

Lately, I have thought much about $\frac{e^{2}}{\hbar c}$.. If only for fun, ... I

write you the equation $\frac{e^{2}}{\hbar c}=\frac{\pi}{2^{4} 3^{3}}$ that occurred to me while playing with $\frac{e^{2}}{\hbar c}$.

Thus, it is appropriate for the constant to be named the Lunn-Heisenberg constant.

Its defect, as we see by comparing formulas (6) and (2), is that it is not accurate. However, we will demonstrate below that a very interesting observation can be made about its reciprocal $\frac{6^{3}}{\pi / 2}$ that further strengthens the connection between Kabbalah and the fine structure constant, and the inaccuracy can be lessened by means of a replacement for $\pi$, as we describe next.

The physicist James Gilson states (2012) that $\pi$ can be replaced or generalized by the perimeter of a regular polygon. He refers to this 
as "quantization" (of $\pi$ ) and comments that motion around a polygon must occur with directional jumps, whereas motion round a circle can take place classically with a continuously changing direction of motion.

Below we will demonstrate that if $\pi$ is "quantized" in this way by means of a 31-sided regular polygon that circumscribes a circle or a 22-sided regular polygon that equalizes the area of a disk, then the Lunn-Heisenberg constant obtains a closer approximation to the value of the fine structure constant. Furthermore, if a circle is approximated by a semi-regular 36-sided polygon which incorporates the ratio 2:5 (or 9:5), as will be explained below, then this results in a significantly better approximation to the fine structure constant. Thus, we suggest the following formula for $\alpha$ :

$$
\alpha \approx \frac{1}{6^{3}}(4 \sin (\pi / 18)+5 \tan (\pi / 18))=0.0072973500657 \ldots 4
$$

The corresponding value for the reciprocal is

$$
\alpha^{-1} \approx 137.03604609684 \ldots
$$

These values are almost within the estimated uncertainty of the values given in formulas (4) and (5), and the value of $\alpha$ in formula (7) varies from the CODATA value given in formula (2) by $0.000035 \%$ and so is well within the observed cosmic variation of $\alpha$ and comparable to the Oklo natural fission reactor measurements mentioned above.

It is a fact that there is not a small probability of discovering a number that approximates $\alpha^{-1}$ to one part in a million by playing with (a root of) a product of integral powers of $2,3,5$, and $\pi$, for example (Peres, 1971). However, such numbers may be difficult to find except by means of an exhaustive computer search. The inclusion of trigonometric ratios allows for the possibility of finding more accurate approximations. Formula (7) approximates $\alpha^{-1}$ to one half part in ten million (by comparison of formulas (8) and (3)) and is discovered by means of a geometric construction.

The next section is an overview of the discovery of the fine structure constant in the 2oth century and a summary of some recent developments. 


\section{THE DISCOVERY OF THE FINE STRUCTURE CONSTANT}

In 1915 Neils Bohr attempted to incorporate relativity into his theory of the atom (Bohr, 1913). Based on his work, in 1916 Arnold Sommerfeld derived the formula (Sommerfeld, 1915, 1916)

$$
E_{n, k}=\frac{Z^{2}}{n^{2}}\left\{1+\left(\frac{e^{2}}{h c}\right)^{2}\left[\frac{n}{k}-\frac{3}{4}\right]\right\}\left(2.7 \times 10^{-11} \mathrm{ergs}\right)
$$

for the orbital energy levels of an electron in the outermost shell of an atom. (This is actually the first order approximation of a more complicated formula that he derived.) Here $k$ and $n$ are quantum numbers that indicate the possible orbits of electrons; $Z$ is the number of protons in the nucleus. The interesting property of the ratio in formula $(1)$ is that it is dimensionless, i.e. the units of $e, h$, and $c$ cancel out (see Born, 1935, p. 539), and it is a "human-sized" number (approximately $1 / 137$ ) while the factors $c, e$, and $h$ are either very large or very small. Also, the relative smallness of the ratio, i.e. $\alpha$ « 1 , allows the use of perturbation methods in Quantum Electrodynamics using power series in $\alpha$. Its significance in formula (9) is to set the scale of the splitting of the spectral lines that are emitted by an atom when it is illuminated by light. For this reason, Sommerfeld named it the fine structure constant. His colleague Friedrich Paschen experimentally measured its value to be approximately 0.00725 . The physicist Arthur Haas, speaking on behalf of the physics community a few years later, stated that Paschen's measurement "implies a brilliant success not only of the ideas of Bohr and Sommerfeld but also of the theory of relativity" (Haas, 1924, p. 45).

The fine structure constant can be conceptualized or interpreted in different ways: for example, as $\left(e^{2} / h\right) / c$ it is the ratio speed of the electron in the ground state of Bohr's hydrogen atom model to the speed of light; as $\frac{\left(e^{2} / r\right) \times(r / c)}{h}$ where two elementary particles are separated by distance $r$, it is the ratio of the electrical action to the quantum action; and as $\frac{e^{2} / m c^{2}}{\hbar / m c}$, where $m$ is the mass of the electron, it is equal to the classical electron radius divided by its reduced Compton wavelength (see Born, 1935; Gilson, 1996; Sherbon, 2014). Furthermore, $\alpha$ can be regarded as the electromagnetic coupling constant because it determines the strength of the interaction between electrons and photons at low energies 
(there are another three coupling constants referring to the weak, strong, and gravitational interactions) (Krag, 2003, p. 396). It also determines the order of magnitude of all nuclear motions, for instance velocities of gas molecules and vibrations of crystal lattices (Born, 1935, p. 357).

In 1925 the Liverpool physicist James Rice attempted to link in a single formula the fine structure constant to cosmic quantities such as the radius of the universe, the electromagnetic radius of the electron, and the gravitational radius of the electron (Rice, 1925).

Confidence in Sommerfeld's formula was strengthened after it was shown to result from Paul Dirac's derivation in 1928 of the linear relativistic wave equation for an electron (Krag, 2003, pp. 407-408). Werner Heisenberg (1934) stated that

... a contradiction-free union of the conditions of quantum theory with the corresponding predictions of field theory is only possible in a theory that provides a particular value for Sommerfeld's constant.

Arthur Eddington caused a stir with a pair of papers (1929, 1931), respectively, in which he claimed that $\alpha^{-1}$ could be calculated by counting the number of states of a pair of electrons:

$$
\alpha^{-1}=16+\frac{1}{2} 16(16-1)+1=137 \text {. }
$$

His contemporaries found his arguments obscure; however, he received support from some physicists, including Pascual Jordan who agreed that it should follow from a complete understanding of the laws of quanta and relativity that the fine structure constant has just the value it has (Jordan, 1937). Max Born (1935) made the same argument, in which he stated that

A perfect theory should be able to derive the number $\alpha$ by purely mathematical reasoning.

Furthermore, he believed that the problem of the proton-to-electron mass $\frac{M}{m} \approx 1840$ was somehow connected to $\alpha$.

In 1948 Julian Schwinger proposed that the cause of the 
discrepancy between the predicted value of the magnetic moment of the electron and experimental data was the result of interactions of the electron with quantum fluctuations of the vacuum. By calculation of these interactions he proposed multiplying the magnetic moment by a

g-factor which had the value $\frac{\alpha}{2 \pi}$ to produce a corrected value known as the anomalous magnetic moment of the electron. In 2017 a semiclassical correction of the magnetic moment of the electron by Oliver Consa involving toroidal solenoid geometry gave a slightly improved g-value of $\sqrt{1+\frac{\alpha}{\pi}}$ (Consa, 2018).

Regarding the value of $\alpha$, Richard Feynman famously stated (1988):

It has been a mystery ever since it was discovered more than fifty years ago, and all good physicists put this number up on their wall and worry about it...

It is impossible to give an account here of all of the proposed formulas for $\alpha$ that have appeared in the literature. Some recent formulas are discussed by Gilson (1997), which presents his own formula

$$
\alpha=\frac{29 \cos (\pi / 137) \tan (\pi /(139 \times 29))}{\pi}=0.00729735253186 \ldots
$$

which is probably to date the most accurate formula and it is calculated from a model of atomic physics and relativity. This formula is difficult to interpret, however, and the numbers $n_{1}=137$ and $n_{2}=29$ have to be selected as the best input into the formula $\alpha\left(n_{1}, n_{2}\right)=n_{2} \cos \left(\pi / n_{1}\right) \tan (\pi$ $\left./\left(n_{1} \times n_{2}\right)\right) / \pi$ where $n_{1}$ and $n_{2}$ are integers.

For Schönfeld and Wilde (2008, 2012), the formula

$$
\frac{1}{\alpha}=\pi \sqrt{2} \frac{m_{q m}}{m_{0}}=137.035999252
$$

is derived in terms of the rest mass $m_{\circ}$ of the electron and its quantummechanical fraction $m_{q m}$.

Gorelik (2009) and Onstott (2019) have compiled formulas for $\alpha$ or $\alpha^{-1}$. The latter includes the formula discovered by W. A. Harrington in 2015.

$$
\alpha^{-1}=16(e-1)^{4}-\sqrt{2(e-1)^{2}}=137.045368 \ldots
$$




\section{NUMBERS AND MYSTICISM}

\section{Mysticism in Philosophy and Physics}

According to the Stanford Encyclopedia of Philosophy (Gellman, 2014), mysticism would, in general, best be thought of as a constellation of distinctive practices, discourses, texts, institutions, traditions, and experiences aimed at human transformation, variously defined in different traditions. A mystical experience is a (purportedly) super senseperceptual or sub sense-perceptual experience granting acquaintance of realities or states of affairs that are of a kind not accessible by way of sense perception, somatosensory modalities, or standard introspection. An example would be an awareness of God (a reality) affording an awareness of one's utter dependence on God (a state of affairs).

In the ancient world mystics were those initiated in the mystery religions. Indeed, the term mysticism is originally derived from the Greek word $\mu v \omega$ meaning "to conceal."

Relating to mysticism in modern times is the view that consciousness imposes "downward causation," i.e. mental events can act to cause physical events, an idea introduced by the social scientist Donald T. Campbell (1974) and developed by the neuropsychologist Roger Sperry (1987). More generally, downward causation describes a causal relationship from higher levels of a system to lower-level parts of that system. This is opposed to naturalism, the idea or belief that only natural (as opposed to supernatural or spiritual) laws and forces operate in the world.

With the discovery of the energy-matter equivalence by Albert Einstein, wave-particle duality by the pioneers of quantum mechanics, and non-locality (quantum entanglement), some physicists have been led to descriptions of the physical world that contain elements and concepts of Eastern mysticism. For example, the 1975 book by physicist Fritjof Capra The Tao of Physics: An Exploration of the Parallels Between Modern Physics and Eastern Mysticism was a bestseller and published in 23 languages. In a video made in the 1980s (Koole, 1986), Capra says

In deep meditative experience the mystics also say that the observer and observed fuse; they can no longer be separated. But they go further: They go to the point where there is no distinction. The entire being dissolves into the universe... 
Later in the video he mentions that he had shown his manuscript of The Tao of Physics in 1973 to Werner Heisenberg, who said that he was well aware of the parallels and open to them. Furthermore, Heisenberg told him that when he had been a guest of the famous poet and philosopher Rabindranath Tagore in India, Tagore had told him that Indian culture was built on the premises of indeterminacy, relativity, interconnectedness, and the dynamic nature of the world, the very worldview that was emerging in quantum physics.

The physicist David Bohm was a proponent of the fundamental idea that beyond the visible, tangible world there lies a deeper, implicate order of undivided wholeness. Moreover, he proposed that the whole universe can be thought of as a flowing hologram, or holomovement, in which a total order is contained, in some implicit sense, in each region of space and time (Bohm, 1980).

\section{Kabbalah and Number Mysticism}

Kabbalah is a Jewish branch of mysticism that emerged in 12th-century Europe and is based on the Zohar, a collection of written, mystical commentaries on the Torah (Scholem, 1990). The aim of Kabbalah is to unite the individual with God.

Number mysticism, which holds the view that numbers have not only a quantitative meaning, but also possess an inherent quality independent of their numerical value is a component of Kabbalah because numbers are associated with the letters of the Hebrew alphabet. The practice of assigning numbers to words is known as gematria (Miller, 2009, p. 258).

The connection between the fine structure constant and Kabbalah was discovered in the 1950 shen the scholar of Jewish mysticism Gershom Scholem pointed out that the numerical values of the letters of the word "Kabbalah" in Hebrew add up to 137 (i.e. $5+30+$ $2+100$ ). Furthermore, as explained by Miller (2009, p. 258), two key words in Kabbalah are "wisdom," which has a numerical value of 73 , and "prophecy," which has a numerical value of 64 , and $73+64=137$. God himself is One. The letters of the word "wheel" in Hebrew also add up to 137. The number 72 is also important in Judaism because the three consecutive verses from Exodus 14:19-21 each contain 72 Hebrew 
letters which can be arranged as 72 triplets of letters (a total of 216 letters), with the order of the letters in the middle set reversed, to form the 72 names of God (Wisnefsky, 1993).

Western number mysticism leads back to Pythagoras. According to him, number is the archetype of all things (Robertson, 1995, p. 96). To the ancient Greek philosophers, archetypes were basic forms that existed on the spiritual plane prior to shaping everything that came into material existence. In the Pythagorean philosophy, number is the mediator between the divine and the earthly and so operations performed with numbers can affect the things related to them. The followers of Pythagoras initiated the sciences of geometry and number theory with their study of polygons, the proof of the Pythagorean Theorem, and investigations of triangular, square, and perfect numbers (numbers that are the sum of their divisors).

The 2oth-century philosopher Carl Jung considered archetypes to be psychic patterns in the collective unconscious that we draw from, especially when we are in a dreaming state. He speculated that there was a unitary reality - the unus mundus - that underlay both mind and matter, and that the primary archetypes of this reality were the simple counting numbers (Robertson, 1995, p. 280).

Any discussion of mysticism would be incomplete without the introduction of the golden ratio and the Fibonacci sequence. Scott Olsen eloquently states in the introduction of his book on the Golden Section (Olsen, 2006) that there is

a secretive tradition that centers on a study of number, harmony, geometry, and cosmology that stretches back through the mists of time into Egyptian, Babylonian, Indian, and Chinese cultures,

and that

the great Pythagorean philosopher, Plato, in his writings and oral teachings, hinted, though enigmatically, that there was a golden key unifying the mysteries.

Therefore, if 137 is truly a mystical number it should have a deep connection to the golden ratio and the Fibonacci sequence. 


\section{The World Clock Dream}

In his long relationship with the physicist Wolfgang Pauli, Jung interpreted many of Pauli's dreams, including the famous dream of the World Clock, which involves a vertical and a horizontal disk having a common center and supported by a black bird. Surrounding both discs is a golden ring. Each disk has 32 segments and on the horizontal disk stands four little men, each holding a pendulum. This is illustrated by Várlaki and Nádai (2008), who posited that this entire configuration represents an algebraic archetype from which, by means of an analysis of the temporal and spatial structures, it is possible to derive the formula

$$
1+2 \times 32+2 \times(32+4)=1+64+72=137 .
$$

They also regard as especially important the formula

$$
\alpha^{-1} \approx 4 \pi^{3}+\pi^{2}+\pi=137.036303776
$$

which was discovered along with some other formulas possibly relating elementary particle masses to the number $\pi$ by a factory worker in the United Kingdom in 1983 (Stanbury, 1983). The reason for this is that the number $4 \pi^{3}+\pi^{2}+\pi$ can be interpreted as being symbolically isomorphic to three power-like rhythms and the four space-like quaternio of the dream.

In mythology birds are a symbol of spiritual transcendence (Jung, 1964, pp. 161-157). Várlaki and Nádai (2008, p. 86) mention that in the Pauli-Jung correspondence the black bird represents the female symbolic figure of anima or wisdom goddess, which is suited to the number 7 .

As a correction to formula (13), the formula

$$
\begin{gathered}
\alpha^{-1} \approx 4 \pi^{3}+\pi^{2}+\pi+\frac{\delta_{x}}{1-\delta_{x}} \frac{\delta_{x}}{1+\delta_{x}}=137.035999066 \ldots \\
\text { where } \delta_{x}=\frac{2 \pi}{x} \text { and } x=360
\end{gathered}
$$

is presented by Várlaki et al. (2009a). The correctional term $\frac{\delta_{x}}{1-\delta_{x}} \frac{\delta_{x}}{1+\delta_{x}}$ 
is a symbol of the two disks that are orthogonal to each other in the dream. Relating to this, Várlaki and Rudas (2009) introduce and discuss the twin concept. This involves complementary relationships in a selfregulating system, for example backward and forward, past and future, or positive and negative.

As an attempt to explain the "many-valuedness" of $\alpha$ suggested by formulas (2) and (4), it is suggested by Várlaki et al. (2009a) that the fine structure constant can be treated as a fuzzy number archetype. Fuzzy sets and fuzzy logic were introduced into physics in order to present a model of Euclidean space-time in which at scales less than a certain length the notion of a point does not exist (Madore, 1992). A fuzzy number is a generalization of an ordinary real number in the sense that it does not refer to one single value but rather to a connected set of possible values, where each possible value has its own weight between $O$ and 1 determined by a membership function. A helpful introduction to this topic was written by Del Castillo-Mussot and Dias (1993).

\section{The Lunn-Heisenberg Constant and Base Six}

In base 6 arithmetic $\frac{1}{\pi} \approx 0.15243102213 .{ }^{5}$ By substituting this in the reciprocal of the Lunn-Heisenberg constant, we obtain

$$
\begin{aligned}
\alpha^{-1} \approx \frac{2 \cdot 6^{3}}{\pi} & =2.6^{3} \cdot 0.15243102213_{6} \\
& =2 \cdot 6^{3}\left(\frac{1}{6}+\frac{5}{6^{2}}+\frac{2}{6^{3}}+\frac{4}{6^{4}}+\frac{3}{6^{5}}+\frac{1}{6^{6}}+\frac{0}{6^{7}}+\frac{2}{6^{8}}+\frac{2}{6^{9}}+\ldots\right) \\
& =72+60+4+\frac{4}{3}+\frac{1}{6}+\frac{1}{108}+0+\frac{1}{1944}+\frac{1}{11664} \ldots
\end{aligned}
$$

Alternatively, if $\pi$ is replaced by the quantized value $\pi_{2 / 5,18}^{\alpha}$, which we will define in formula (34) below, then (also in base 6)

$$
\begin{aligned}
\alpha^{-1} \approx \frac{2 \cdot 6^{3}}{\pi_{2 / 5,18}^{\alpha}} & =2.6^{3} \cdot 0.15230352051_{6} \\
& =2 \cdot 6^{3}\left(\frac{1}{6}+\frac{5}{6^{2}}+\frac{2}{6^{3}}+\frac{3}{6^{4}}+\frac{0}{6^{5}}+\frac{3}{6^{6}}+\frac{5}{6^{7}}+\frac{2}{6^{8}}+\ldots\right) \\
& =72+60+4+1+0+\frac{1}{36}+\frac{5}{648}+\frac{1}{1944} \ldots
\end{aligned}
$$


This calculation generates the Kabbalistic formula

$$
72+60+4+1=73+64=137
$$

that was mentioned above. It also matches formula (12) resulting from the interpretation of the World Clock Dream by Várlaki et al.

\section{The Number 137 as a Code for the Fibonacci Sequence}

The Fibonacci sequence, celebrated for its ubiquity in mathematics, science, and nature, is the infinite sequence of integers

$$
0,1,1,2,3,5,8,13,21,34,55,89,144,233,377, \ldots
$$

which is characterized by the property that every digit in the sequence is the sum of the preceding two digits. It is named after the Italian mathematician Leonardo of Pisa, known as Fibonacci. In his book Liber Abaci published in 1202 he introduced this sequence to mathematics in Western Europe. It is an important fact that the sequence of ratios of successive Fibonacci numbers

$$
\frac{2}{1}, \frac{3}{2}, \frac{5}{3}, \frac{8}{5}, \frac{13}{8}, \frac{21}{13}, \frac{34}{21}, \ldots
$$

converges to the golden ratio $\frac{1+\sqrt{5}}{2}$.

To explain the connection between the Fibonacci sequence and the number 137 we first observe that its expression in base 6 is

$$
345_{6}=3 \times 6^{2}+4 \times 6+5=108+24+5=137 .
$$

The number 5 , as the fifth non-zero element of the Fibonacci sequence, can be regarded as a reference to the Fibonacci sequence. To see how the numbers 108 and 24 relate to the Fibonacci sequence we will look at the sequence that can be derived from it by calculating the digital roots of the numbers of the sequence.

The digital root or repeated digital sum of a natural number is the single digit obtained by an iterative process of summing the digits (of the number) and, on each iteration, using the result from the previous 
iteration until a single-digit number is reached. For example, the digital root of 11664 is 9 because $1+1+6+6+4=18$ and $1+8=9$. If the digital root of a number $n$ is the number $p$, then $n-p$ is a multiple of 9 and (in modular arithmetic) $n \equiv p(\bmod 9)$ (see Wikipedia, 2016). Therefore, the number 9 behaves like " $\mathrm{o}$ " in the calculation of the digital root of a number. This is the basis of the method of casting out nines (see Wikipedia, n.d.). For example, the number 729735 has the same digital root as 735 .

If we calculate the digital roots of the numbers of the Fibonacci sequence, we obtain the sequence

$$
\text { (o), 1, 1, 2, 3, 5, 8, 4, 3, 7, 1, 8, 9, 8, 8, 7, 6, 4, 1, 5, 6, 2, 8, 1, (9), 1, 1, . . }
$$

which is a repeating sequence of digits, beginning with a (9) on the first repetition and subsequent repetitions (see Wikipedia, 2016). There are 24 digits in each repeating string, and (as mentioned in Leeds, 2013) the sum of the 24 digits in the first string (starting with 0 ) is 108.

\section{Pentagons, Pythagorean Triples, and the Golden Ratio}

There is a connection between the two formulas (15) and (18) and the geometry of a pentagon: The angles at the vertices of a pentagon are $108^{\circ}$ and the diagonals intersect at angles of $108^{\circ}$ and $72^{\circ}$. Furthermore, the two diagonals drawn from any vertex trisect the angle at the vertex into three angles which are each equal to $36^{\circ}$.

The number 6 is a perfect number and a triangular number and the number 36 is also a triangular number obtained from the Greek tetractys (a triangular figure consisting of ten points arranged in four rows) by doubling the number of points on each side. For this and many other reasons (Keppler et al., 1997, pp. 133-136), the number 36 was revered by the Pythagoreans. The 36 th triangular number is the occult number 666.

The numbers $36,60,72$, and 132 also have a connection in the existence of certain regular polyhedra constructed only from pentagons having so many faces (Lanzi, 2017; RobertLovesPi, 2016, 2015; Weisstein, 1999). ${ }^{6}$

The expression $345_{6}$ of 137 in base 6 (i.e. formula (18)) is suggestive of 
a connection between the fine structure constant and the Pythagorean Theorem and Pythagorean triples, in particular the triple $(3,4,5)$. (If any three natural numbers $a, b, c$ satisfy the property of the Pythagorean Theorem, i.e. $a^{2}+b^{2}=c^{2}$, then they form the sides of a right triangle and they are called a Pythagorean triple.) It is a fact that in every Pythagorean triple $(a, b, c)$, the number 3 is always a factor of exactly one of $a$ and $b$, the number 4 is always a factor of exactly one of $a$ and $b$, the number 5 is always a factor of exactly one of the three numbers $a, b$, and $c$, and the largest number that always divides $a \cdot b \cdot c$ is 60 (see Wikipedia, 2018).

It is well-known (Bogomolny, 1996) that the golden ratio appears as the ratio of a diagonal to a side of a regular pentagon. The golden ratio is also expressed elegantly in the geometry of a 3-4-5 right triangle (Brown, 2016, p. 327). We mention also the interesting formula

$$
3^{3}+4^{3}+5^{3}=6^{3}
$$

which gives a connection between the number $6^{3}=216$ and the triple $(3,4,5)$.

The author Bonnie Gaunt has discovered many surprising connections between the gematria of phrases and names in the bible (in their Greek or Hebrew spelling), astronomical data (including planetary and solar geometry), and the 3-4-5 right triangle, in particular. For example, she explains that the gematria of "Lord Jesus Christ" in its Greek spelling is the number 3168, and a 3-4-5 right triangle with hypotenuse equal to the radius of the Earth (3960 miles) has one side of length 3168 miles (Gaunt, 2000).

\section{The Significance of 108 in Religion, Mysticism, and Astronomy}

The number 108 is considered sacred by the Dharmic Religions, such as Hinduism, Buddhism, and Jainism (Wikipedia, 2017). Traditionally, malas, used for counting the repetitions of a mantra, come as a string of 108 beads (plus the "guru bead").

As explained by Margaret Starbird (2003), the numbers 108 and 1080 represent the Sacred Feminine in Christianity. This is a consequence of the esoteric tradition of sacred number, geometry, and gematria having passed into Christianity through the mystery schools of 
the Hellenized world. The number 1080 was associated with the moon because it approximated the radius of the moon when measured in miles. In the Greek method of gematria, the letters of the words "Holy Spirit" and "Earth Spirit," which are anagrams in their Greek spelling, add up to 1080. Also, the spelling of "dove" in Greek has a gematria of 801. For this reason, the Holy Spirit and Earth Spirit are both linked by gematria to the lunar/feminine principle and the dove. Furthermore, the value the first Greek letter alpha is 1, and the value of the last Greek letter omega is 800 . Their sum 801 is therefore associated with the "Alpha and Omega," an epithet of the "Holy One" which represents the eternal union of all opposites.

Michell (2008, p. 209) mentions that the words Prophecy and Wisdom in their Greek spelling both have a gematria of 1080, thus giving these two words another connection to the number 137 and the fine structure constant.

H. Spencer Lewis (1979) mentions an ancient and mysterious tradition of unknown origin that requires a periodicity of active and inactive cycles of the Rosicrucian Order, each of which lasts for 108 years. He also mentions that the number 108 is significant in itself to all occult students.

In astronomy, the distance between the Earth and the sun is approximately 108 times the diameter of the sun, and the distance between the Earth and the moon is approximately 108 times the diameter of the moon (this is the reason the moon precisely covers the sun during a total eclipse). What's more, the diameter of the sun is approximately 108 times the diameter of the Earth.

There is an interesting discussion about possible reasons for the importance of 108 from Koenraad Elst (2003).

\section{Plato's Mystical Numbers}

Two other numbers of interest relating to formula (18) are the products $24 \times 108=2592$ and $5 \times 24 \times 108=12960$. The former relates to the Platonic Year or Great Year (25,920 years), which is the amount of time (according to Plato) required for a complete precession of the equinoxes (Barton, 1908), i.e. an ecliptical year, and the latter relates to the number $12,960,000$ that is widely believed to be Plato's Nuptial 
Number or Sovereign Number, which he introduces in a passage in book VIII of the Republic, by means of a lengthy and convoluted description, as a number that controls the formation of the world (Barton, 1908).

\section{THE QUANTIZATION OF $\pi$}

As mentioned above, we will explore what happens when we replace the numerator $\pi$ in the Lunn-Heisenberg constant by certain approximations (i.e. quantizations) of $\pi$. Readers who are less mathematically inclined may skip over this section.

As preparation for this we will first review the problem of calculating $\pi$ historically. In classical antiquity there were essentially two different methods used to find an approximation to $\pi$ that may conveniently be referred to here as the Greek method (see Beckman, 1971, pp. 64-67) and the Egyptian method (see Beckman, 1971, pp. 24-25). ${ }^{8}$

\section{The Approximation of $\pi$ (The Greek Method)}

The first scientific engineer, Archimedes of Syracuse (born in 287 BC), described his method of approximating $\pi$ in his treatise On the Measurement of a Circle. In this he demonstrated that a circle can be closely approximated arbitrarily by an inscribed or circumscribed regular polygon, as in Figure 1 below with inscribed and circumscribed hexagons.

If the radius of the circle is 1 , then the areas of the inscribed and circumscribed hexagons are $3 \sin \pi / 3$ and $6 \tan \pi / 6$, respectively, and the corresponding perimeters are $12 \sin \pi / 6$ and $12 \tan \pi / 6$, respectively. The corresponding approximations to $\pi$ determined by the inscribed hexagon are

$$
\begin{aligned}
& \pi \approx 3 \sin \pi / 3=2.598 \ldots \text { (by comparison of areas) } \\
& \pi \approx 6 \sin \pi / 6=3 \quad \text { (by comparison of perimeters). }
\end{aligned}
$$

For the circumscribed hexagon, the area and perimeter approximations both determine that $\pi \approx 6 \tan (\pi / 6)=2 \sqrt{3} \approx 3.4641 \ldots$

Archimedes determined that $3.14085 \approx 223 / 71<\pi<22 / 7 \approx 3.14286$ by performing calculations relating to inscribed and circumscribed polygons with 96 sides. 


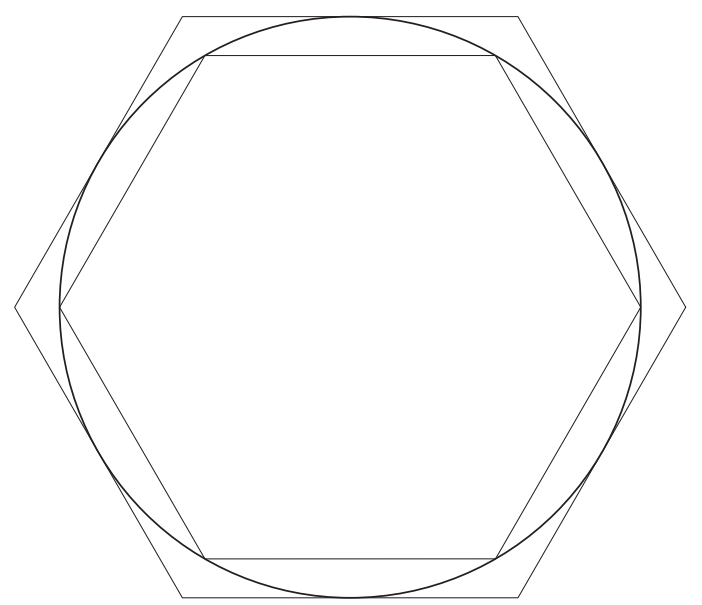

Figure 1. The Greek method of calculating $\pi$.

For a circumscribed polygon with an unspecified number $(n)$ of sides, the formula above generalizes to

$$
\pi \approx n \tan (\pi / n) \text {. }
$$

A variation of the Archimedean method is to approximate a circle by a polygon which has the same area as the disk enclosed by the circle. For example, the area of the inscribed hexagon above is $3 \sin (\pi / 3)$. Therefore, the radius of the circle that contains the same area is

$\sqrt{\frac{\sin (\pi / 3)}{\pi / 3}}$. In this case the hexagon is area equalizing (for the circle with this radius) and the circle interpolates the hexagon. Its perimeter (as calculated above) is $12 \sin \pi / 6$. By comparison of the perimeter of the hexagon with the circumference of the interpolating circle we have

or

$$
12 \sin (\pi / 6) \approx 2 \pi \sin \sqrt{\frac{\sin (\pi / 3)}{\pi / 3}}
$$

$$
\pi \approx \frac{6 \sin \left(\frac{\pi}{6}\right)}{\sqrt{\frac{\sin (\pi / 3)}{\pi / 3}}}=3.2989 \ldots
$$


The general formula for approximating $\pi$ by means of an area equalizing polygon with $n$ sides is

$$
\pi \approx \frac{n \sin (\pi / n)}{\sqrt{\frac{\sin (2 \pi / n)}{2 \pi / n}}}
$$

An alternative is to approximate a circle by a polygon which is perimeter equalizing. It is left to the reader to check that the approximation to $\pi$ which results from this is the same as formula (20).

\section{The Approximation of $\pi$ (the Egyptian Method)}

The Egyptian method is described by Ahmes in a papyrus scroll found in Thebes and acquired by the Scottish Antiquary Henry Rhind in 1858. It is now mostly displayed in the British Museum. A part which was discovered in 1922 is now displayed in the Brooklyn Museum. In problem no. 50 in the papyrus, Ahmes describes the approximation of a disk by an octagon formed from a grid of squares, as shown in Figure 2.

The octagon in this case can be referred to as a semi-regular rather than a regular octagon because sides of the same length alternate with sides of a different length.

The circle has a diameter of 9 units and each square has a sidelength of 3 units. Therefore, the area of the disk $\pi\left(\frac{9^{2}}{2}\right)$ is approximately equal to the area of seven squares, which is $7(3)^{2}=63$. Ahmes preferred the number 64 , which is the area of a square of side-length 8 . Thus, he arrived at $\pi \approx 4 \times\left(\frac{8}{9}\right)^{2}=3.16049 \ldots$.

Another way to calculate an approximate value of $\pi$ from the same diagram is to use the perimeter $4(3)+4(3 \sqrt{2})$ of the octagon as an approximation of the circumference $2 \pi\left(\frac{9}{2}\right)$ of the circle. This produces $\pi \approx \frac{4(1+\sqrt{2})}{3}=3.219$.

Furthermore, it is possible to generalize the construction by replacing the segments of the octagon of length 3 contained in the sides of the surrounding square by segments of a different length (labeled $x$ in Figure 3) centered in each side of the square. 


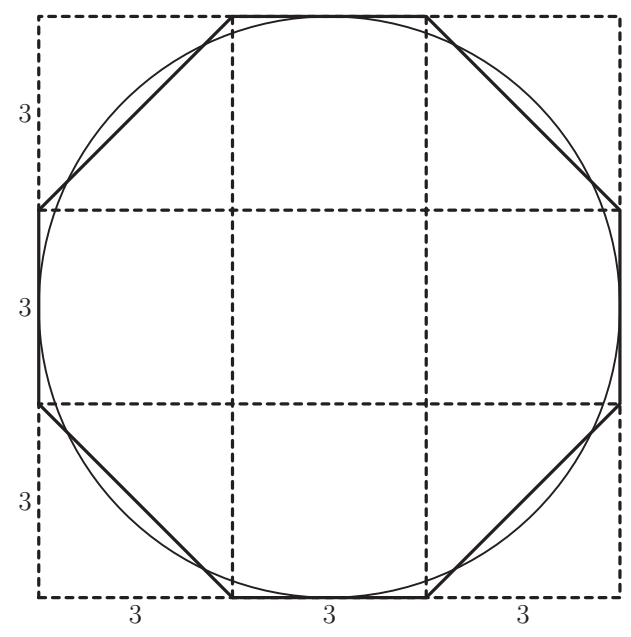

Figure 2. The Egyptian method of calculating $\pi$.

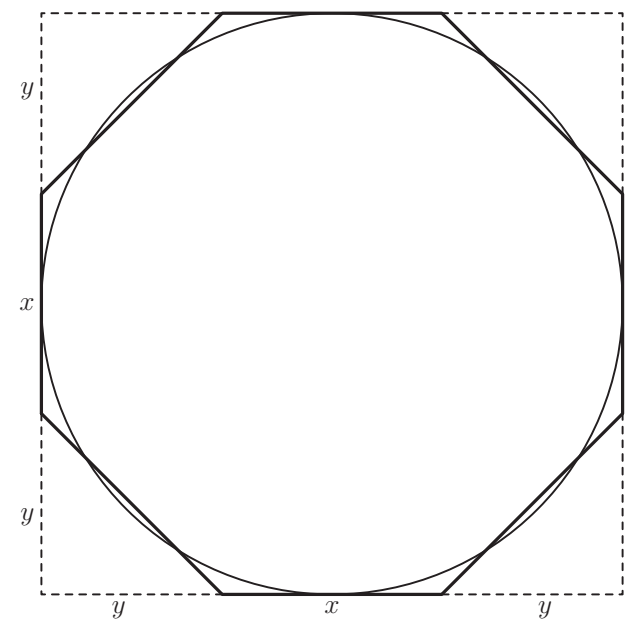

Figure 3. A generalization of the Egyptian method.

If we work with a diameter of 2 (instead of 9) for the circle, then $x$ $+2 y=2$ and the area of the octagon is $x^{2}+4 x y+2 y^{2}$ or $2+2 x-\frac{x^{2}}{2}$ and its perimeter is $4(x+\sqrt{2})$ or $2(2-\sqrt{2}) x+\sqrt{2}$. Therefore, there are the following two possible approximations for $\pi$ for any value of $x$ between 0 and 2: 


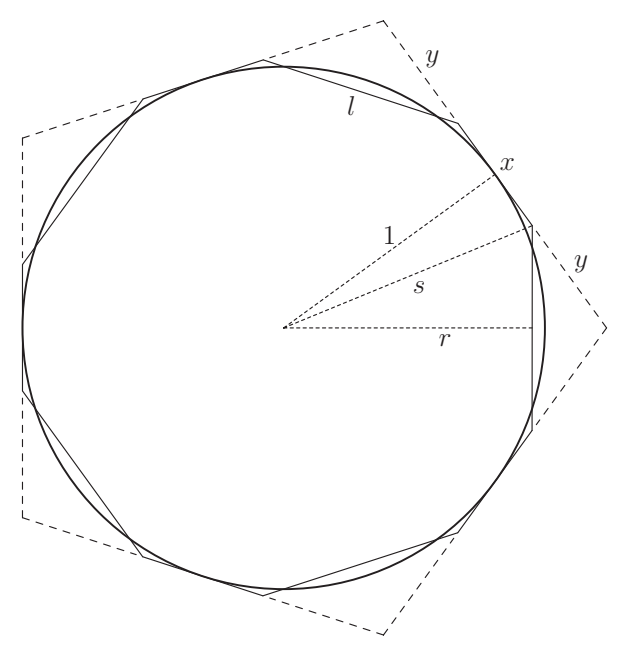

Figure 4. Replacing a square by a pentagon.

$$
\begin{gathered}
\pi \approx 2+2 x-\frac{x^{2}}{2} \quad \text { (by comparison of areas) } \\
\pi \approx(2-\sqrt{2}) x+2 \sqrt{2} \text { (by comparison of perimeters). }
\end{gathered}
$$

Ahmes' construction corresponds to $x=2 / 3$. If $x=9 / 17$, for example, then the second formula above gives $\pi \approx 3.1385$.

Ahmes' construction can be generalized further by replacing the surrounding square by any other regular polygon. The diagram in Figure 4 shows the construction with the square replaced by a regular pentagon to produce a semi-regular decagon.

The symbols $I, r$, and $s$ were inserted in order to facilitate the following calculation of the area and perimeter of the semi-regular decagon, which we will denote as $A_{5}(x)$ and $P_{5}(x)$, respectively. (We assume that the pentagon circumscribes a circle of radius 1.) Using trigonometry and the properties of right triangles,

and

$$
\begin{gathered}
\frac{x}{2}+y=\tan \frac{\pi}{5}, \\
r^{2}+\left(\frac{l}{2}\right)^{2}=s^{2}=1+\left(\frac{x}{2}\right)^{2}
\end{gathered}
$$

$$
l^{2}=2 y^{2}\left(1+\cos \frac{2 \pi}{5}\right)=4 y^{2} \cos ^{2} \frac{\pi}{5} \text {. }
$$


Therefore

$$
\begin{gathered}
A_{5}(x)=5\left(\frac{x}{2}+\frac{r l}{2}\right) \\
=\frac{5}{2}\left(x+y \cos \frac{\pi}{5} \sqrt{4+x^{2}-4 y^{2} \cos ^{2} \frac{\pi}{5}}\right) \\
=\frac{5}{2}\left(x+\left(\sin \frac{\pi}{5}-\frac{x}{2} \cos \frac{\pi}{5}\right) \sqrt{x^{2} \sin ^{2} \frac{\pi}{5}+2 x \sin \frac{2 \pi}{5}+4 \cos ^{2} \frac{\pi}{5}}\right)
\end{gathered}
$$

and

$$
\begin{aligned}
P_{5}(x) & =5(x+l) \\
& =5\left(x+2 y \cos \frac{\pi}{5}\right) \\
& =5\left(x\left(1-\cos \frac{\pi}{5}\right)+2 \sin \frac{\pi}{5}\right) .
\end{aligned}
$$

In this case the perimeter formula is much simpler. If we denote the ratio $y / x$ by $\lambda$ then we can derive a formula for the perimeter in terms of $\lambda$, which we will designate as $p_{5}(\lambda)$ : From formula (22) we have

$$
x=\frac{2 \tan \frac{\pi}{5}}{1+2 \lambda} .
$$

If we substitute this in formula (23) we obtain

$$
\begin{aligned}
p_{5}(\lambda)= & 5\left(\frac{2 \tan \frac{\pi}{5}}{1+2 \lambda}\left(1-\cos \frac{\pi}{5}\right)+2 \sin \frac{\pi}{5}\right) \\
& =\frac{5}{1+2 \lambda}\left(2 \tan \frac{\pi}{5}+4 \lambda \sin \frac{\pi}{5}\right) .
\end{aligned}
$$

The corresponding approximation to $\pi$ for any choice of $\lambda$ is

$$
\pi \approx \frac{p_{5}(\lambda)}{2}=\frac{5}{1+2 \lambda}\left(\tan \frac{\pi}{5}+2 \lambda \sin \frac{\pi}{5}\right)
$$

For example, if $\lambda=6 / 5$ then the approximation to $\pi$ is $3.14298 \ldots$.

An identical formula with " 5 " replaced by " $n$ " can be used if the 
surrounding pentagon is replaced by any regular polygon with an unspecified number of sides (n), i.e. formula (24) generalizes to

$$
p_{n}(\lambda)=\frac{n}{(1+2 \lambda)}\left(2 \tan \frac{\pi}{n}+4 \lambda \sin \frac{\pi}{n}\right)
$$

and formula (25) generalizes to

$$
\pi \approx \frac{p_{n}(\lambda)}{2}=\frac{n}{(1+2 \lambda)}\left(\tan \frac{\pi}{n}+2 \lambda \sin \frac{\pi}{n}\right) .
$$

\section{Application to the Lunn-Heisenberg Constant}

We will now apply formulas (20), (21), and (27) in order to modify the Lunn-Heisenberg constant. For convenience, we will denote the righthand side of formula (20) by $\pi_{n}^{c}$ ("c" for circumscribed), the right-hand side of formula (21) by $\pi_{n}^{e}$ ("e" for equalizing), and the right-hand side of formula (27) by $\pi_{\lambda, n}^{a}$ ("a" for Ahmes), i.e.

$$
\begin{aligned}
\pi_{n}^{c} & =n \tan (\pi / n) \\
\pi_{n}^{e} & =\frac{n \sin (\pi / n)}{\sqrt{\frac{n \sin (2 \pi / n)}{2 \pi}}} \\
\pi_{\lambda, n}^{a}=\frac{p_{n}(\lambda)}{2}= & \frac{n}{(1+2 \lambda)}\left(\tan \frac{\pi}{n}+2 \lambda \sin \frac{\pi}{n}\right) .
\end{aligned}
$$

Because formula (6) determines an approximation to $\alpha$ that is too small, we replace $\pi$ by $\pi_{n}^{c}$ according to formula (28) for different choices of $n$ in order to obtain a larger value. Table 1 shows the value of $\frac{\pi_{n}^{c}}{2 \cdot 6^{3}}$ correct to eight decimal positions for a few values of $n$.

The best approximation to the fine structure constant occurs for $n=31$. For values of $n$ less than 31 the ratio $\frac{\pi_{n}^{c}}{2 \cdot 6^{3}}$ is too big, and for values of $n$ larger than 31 it is too small. Therefore

$$
\alpha \approx \frac{\pi_{31}^{c}}{2 \cdot 6^{3}}=\frac{31 \tan (\pi / 31)}{432}=0.007297203437
$$

According to formula (2) this differs from the correct value of $\alpha$ by 1 unit in the $5^{\text {th }}$ significant digit. A 31-sided regular polygon is called a triacontakai-henagon. 
TABLE 1 The Best Area Equalizing Polygon for $n=29-33$

\begin{tabular}{cc}
\hline \hline $\boldsymbol{n}$ & $\pi_{n}^{c} /(\mathbf{2 \times 6})$ \\
\hline 29 & 0.00730079 \\
30 & 0.00729891 \\
31 & 0.00729720 \\
32 & 0.00729566 \\
33 & 0.00729425 \\
\hline
\end{tabular}

TABLE 2 The Best Area Equalizing Polygon for $n=20-24$

\begin{tabular}{cl}
\hline \hline $\boldsymbol{n}$ & $\pi_{n}^{e} /\left(2 \times 6^{3}\right)$ \\
\hline 20 & 0.00730235 \\
21 & 0.00729952 \\
22 & 0.00729708 \\
23 & 0.00729495 \\
24 & 0.00729309 \\
\hline
\end{tabular}

Similarly, we can replace $\pi$ in formula (6) by $\pi_{n}^{e}$ according to formula (29) for different choices of $n$. Table 2 shows the value of $\frac{\pi_{n}^{e}}{2 \cdot 6^{3}}$ correct to eight decimal positions for a few values of $n$.

The best approximation to the fine structure constant occurs for $n=22$. For values of $n$ less than 22 the ratio $\frac{\pi_{n}^{e}}{2 \cdot 6^{3}}$ is too big and for value of $n$ larger than 22 it is too small. Therefore,

$$
\alpha \approx \frac{\pi_{22}^{e}}{2 \cdot 6^{3}}=\frac{11 \sin (\pi / 22)}{216 \sqrt{\frac{11 \sin (\pi / 11)}{\pi}}}=0.007297081436 \ldots
$$

According to formula (2) this differs from the correct value of $\alpha$ by three units in the $5^{\text {th }}$ significant digit. A 22 -sided regular polygon is called an icosikaidigon.

The replacement of $\pi$ in formula (6) by $\pi_{\lambda, n}^{a}$ according to formula (30) for different choices of $n$ is more complicated because of the additional parameter $\lambda$. In fact, it is possible (for each fixed value of $n$ ) to find (numerically) the value of $\lambda$ that is the solution of the equation

$$
\frac{\pi_{\lambda, n}^{\alpha}}{2 \cdot 6^{3}}=0.0072973525664 .
$$

In other words, it is possible (for each fixed value of $n$ ) to adjust the numerator of the fraction on the left-hand side of the equation above 
TABLE 3

Solving for $\lambda$ in Formula (33) for Different Values of $n$

\begin{tabular}{llllll}
\hline \hline $\mathbf{n}$ & \multicolumn{1}{c}{$\lambda$} & \multicolumn{1}{c}{ ratio } & $\mathrm{n}$ & \multicolumn{1}{c}{$\lambda$} & \multicolumn{1}{c}{ ratio } \\
\hline 3 & 1.8432 & $540 / 293$ & 17 & 0.44219 & $241 / 545$ \\
4 & 1.3078 & $310 / 237$ & 18 & 0.39996 & $2 / 5$ \\
5 & 1.1245 & $930 / 827$ & 19 & 0.35937 & $216 / 601$ \\
6 & 1.0221 & $325 / 318$ & 20 & 0.32045 & $9000 / 28081$ \\
7 & 0.94806 & $50000 / 52737$ & 21 & 0.28323 & $177 / 625$ \\
8 & 0.88611 & $459 / 518$ & 22 & 0.24771 & $8000 / 32297$ \\
9 & 0.82986 & $40000 / 48201$ & 23 & 0.21386 & $179 / 837$ \\
10 & 0.77658 & $608 / 783$ & 24 & 0.18166 & $2800 / 1541$ \\
11 & 0.72507 & $12500 / 17239$ & 25 & 0.15106 & $16000 / 10589$ \\
12 & 0.67485 & $687 / 1018$ & 26 & 0.12202 & $3000 / 2459$ \\
13 & 0.62579 & $20000 / 31959$ & 27 & 0.094472 & $41 / 434$ \\
14 & 0.57789 & $523 / 905$ & 28 & 0.068363 & $2500 / 36571$ \\
15 & 0.53125 & $332 / 625$ & 29 & 0.043628 & $25 / 573$ \\
16 & 0.48598 & $434 / 893$ & 30 & 0.020200 & $2000 / 99009$ \\
\hline
\end{tabular}

in order to produce a ratio that equals the currently accepted measured value of the fine structure constant. The question is whether there is something interesting about the value of $\lambda$ obtained in this way (for each $n$ ). This question will be answered with reference to Table 3 where, for each value of $n$, the value of $\lambda$ which solves formula (33) is given with an accuracy of five significant digits. The inverse symbolic calculator which is available at the website (Singer et al., 2018) was employed to find the corresponding fraction in the third column which matches this value of $\lambda$ with an accuracy of at least four significant digits.

Recall that $n$ determines the number of sides of the regular polygon that circumscribes the circle and $\lambda$ is the ratio according to which a semi-regular polygon with double the number of sides is created in order to approximate the circle more accurately. The table stops at $n$ $=30$ because for larger values of $n$ there is no solution for $\lambda$ that is a positive number. 
The value of $n$ which is very interesting and surprising is $n=18$ because the corresponding value of $\lambda$ is the simple ratio $2 / 5$. The corresponding semi-regular polygon has 36 sides and it can be identified by any of the ratios $y / x=2 / 5,(2 y) / x=4 / 5,(2 y+x) / x=9 / 5$ or their reciprocals. Formula (7) is thus a consequence of setting $n=18$ and $\lambda=$ $\frac{2}{5}$ in formula (30) in order to obtain the expression for $\pi_{2 / 5,18}^{\alpha}$ that replaces $\pi$ in formula (6). We note that

$$
\pi_{2,5,18}^{\alpha}=3.15245522842 \ldots
$$

By means of the following properties of trigonometric ratios

$$
\begin{gathered}
4 \sin \frac{\pi}{18}+5 \tan \frac{\pi}{18}=\tan \frac{\pi}{18}\left(4 \cos \frac{\pi}{18}+5\right) \\
=\frac{1}{\sqrt{3}}\left(1-4 \sin \frac{\pi}{18}\right)\left(4 \cos \frac{\pi}{18}+5\right) \\
=\frac{1}{\sqrt{3}}\left(1-8 \cos \frac{\pi}{36} \sin \frac{\pi}{36}\right)\left(1+8 \cos ^{2} \frac{\pi}{36}\right)
\end{gathered}
$$

(the second step above is explained in the Appendix), we obtain the following alternative formulation of formula (7) which is perhaps more pleasing and involves the angle $\pi / 36.9$

$$
\alpha \approx \frac{1}{6^{3}} \frac{\left(1-8 \cos \left(\frac{\pi}{36}\right) \sin \left(\frac{\pi}{36}\right)\right)\left(1+\cos ^{2}\left(\frac{\pi}{36}\right)\right)}{\sqrt{3}}=0.0072973500657
$$

In summary, we have that

—among circumscribed polygons (or perimeter-equalizing polygons), a 31-sided regular polygon produces the best approximation to the value of the fine structure constant by means of formula (31).

—among area-equalizing polygons, a 22-sided regular polygon produces the best approximation to the value of the fine structure constant by means of formula (32).

-among semi-regular polygons the simplest construction that accurately approximates the fine structure constant (by means of formula (35)) is a 36 -sided semi-regular polygon with a 2:5 ratio. 
We remark that there is a surprising connection between the numbers 22 and 31, which is that 22 is the fourth pentagonal number (defined by the formula $n(3 n-1) / 2)$, and 31 is the fourth centered pentagonal number (defined by the formula $\frac{5 n^{2}-5 n+2}{2}$ ).

\section{THE WORLD CLOCK DREAM REVISITED}

We will now point out some interesting similarities between formula (7) and its Pythagorean geometric derivation involving a 36-sided semiregular polygon and formulas (13) and (14). We will also interpret the occurrence of the numbers $4,5,6$, and 8 in formulas (7) and (35) in the context of the World Clock Dream.

As briefly mentioned earlier, the formulas (13) and (14) are interpreted by Várlaki and Nádai (2008) and Várlaki and Rudas (2009) in terms of unconscious creative background processes (as exemplified by the dreams of Wolfgang Pauli) and number archetypes or archetypal patterns and images. The number $\pi$ is a geometric number. The number 3 arises from the three temporal rhythms in Pauli's World Clock Dream and manifests in formula (13) as $\pi, \pi^{2}$, and $\pi^{3}$. The number 4 arises from four figures representing the four cardinal directions in the dream (and some of his other dreams) and manifests as the coefficient of $\pi^{3}$ in formula (13).

In formula (7) the number 4 also manifests as a coefficient. The three temporal rhythms manifest as $6^{2}$ and $6^{3}$ in formulas (7) and (35).

The number 5 is another coefficient in formula (7). It arises in the World Clock Dream as the sum of the four little men and the black bird.

The number 8, which is a coefficient in formula (35), is represented in the World Clock dream by the four little men, each carrying a pendulum. A pendulum indicates multiplication by 2 . Thus $4 \times 2=8$.

Furthermore, the twin property which manifests in formula (14) in

the corrective term $\frac{\delta_{x}}{1-\delta_{x}} \frac{\delta_{x}}{1+\delta_{x}}$ with $\delta_{x}=\frac{\pi}{360}$ corresponding to the two disks that are orthogonal to each other, also manifests in formula (7) in the two trigonometric terms (sine and tangent), which are defined as the ratios of the sides of a right triangle.

In Pauli's dreams the numbers 32 and 36 are interchangeable because the Swedish physicist, Johannes Rydberg, who discovered the 
formula $2 p^{2}$ (that fascinated Pauli) for the number of elements in each period of the Periodic Table, had initially assumed that there were 36 elements, not 32, in the period containing the rare earths (lanthanides) (Várlaki \& Nádai, 2008, pp. 91-93). If there are 36 instead of 32 equal segments of the horizontal and vertical disks in the World Clock Dream, then the angle of each segment is $\frac{2 \pi}{36}=\frac{\pi}{18}$ radians $\left(\right.$ or $\left.10^{\circ}\right)$, the angle used in formula (7).

Finally, we mention that if the value $x=360$ in formula (14) is replaced by $x=392$, i.e. $\delta_{x}=\frac{2 \pi}{392}$, then the left-hand side evaluates to 137.036046796 , which is very close to the value for $\alpha^{-1}$ in formula (8). This is surprising in view of the fact that if $x=32$, i.e. $\delta_{x}=\frac{2 \pi}{32}$, then the left-hand side of formula (14) evaluates to 136.996 , which is very close to 137 (as pointed out by Várlaki \& Rudas, 2009).

\section{NUMBER 7}

As mentioned above, the number 7 is connected with the black bird as the anima in the World Clock Dream. To the classical Greeks, 7 was the "virgin number" because among the first ten counting numbers it is the only one that is neither a multiple nor a factor of any of the others (Starbird, 2003, p. 22). In an ancient diagram known as "the seed of life" six intersecting circles representing all of physical creation are united in spiritual wholeness by a seventh circle passing though their centers (Starbird, 2003, pp. 26-77). In general, the number 7 is associated with eternity, perfection, and completion (in the spiritual sense) (Starbird, 2003, p. 47).

A natural formula to consider is $137+7=144=12^{2}$. The number 144 is the 12th (non-zero) Fibonacci number. It is associated with fullness or completeness on the Earthly or physical plane. A discussion about the numbers 7 and 12 can be found in Starbird (2003, pp. 48-49). It is also mentioned by Gaunt (2000, p. 144) that the Hebrew word kedem, which is translated as "beginning", "eternal", "origin", or "everlasting", has a numerical value of 144 .

A surprising connection between the numbers 24 and 70 is the identity 


$$
1^{2}+2^{2}+3^{2}+\cdots+23^{2}+24^{2}=70^{2}
$$

(i.e. the sum of the first 24 perfect squares is the perfect square $70^{2}$.) This identity is special because 24 is the only natural number $k$ (besides the trivial case $k=1$ ) in the formula

$$
1^{2}+2^{2}+3^{2}+\cdots+(k-1)^{2}+k^{2}=p^{2}
$$

for which the solution $p$ is a natural number. The physicist and string theorist John Baez explains, in a video on his website about his three favorite numbers (Baez, 2008), why this identity is the reason string theory works best in 26 dimensions. His three favorite numbers are 5 , 8 , and 24. John Michell (2008, p. 65) speculates that the words pnuema (breath) and eagle may have been adopted as religious terms for Spirit because they both have a gematria of $576=24^{2}$ in their Greek spelling. He goes on to mention that the importance of 24 is that it can be expressed as $8+8+8$, and 888 is the gematria of Jesus.

\section{LIGHT, ELECTRIC CHARGE, AND PLANCK'S CONSTANT}

It is possible to speculate about the symbolic meaning of the numbers 108,24 , and 5 in the sum $5+24+108=137$. As mentioned above, the number 24 signifies Spirit, which is associated with light. The number 108 , as the symbol for the Sacred Feminine, is also associated with the Earth and negative charge (Michell, 2008, p. 205). The basic unit of negative charge is the charge of an electron. Finally, it is a curious fact that in astrology the glyph for Saturn resembles number 5 , and also resembles the symbol for Planck's constant. An interesting commentary about this is posted on the Facebook page of the Esoteric Science Institute (Poirier, 2017). Therefore, we have a remarkable correspondence between the numbers 108,24 , and 5 and the quantities $e, c$, and $h$, respectively, which combine to define the fine structure constant.

\section{CONCLUSION}

With our investigation of the Lunn-Heisenberg constant we have expanded on certain speculations of a connection between the fine structure constant and mysticism (especially Pythagorean mysticism and Kabbalah). We have given a new and broader context to the 
methodology of Várlaki et al. in their interpretation of Pauli's World Clock Dream and other dreams. Furthermore, the number 137 also has a surprising connection to the repeating sequence of digital roots of the Fibonacci numbers via the formula $137=345_{6}=108+24+5$.

In summary:

1. Certain numbers important in mathematics and science, for example 137 and 108, are also important in mysticism and world religions.

2. Certain numbers, features, and patterns in the formulas (or derivation of the formulas) that approximate the fine structure constant or calculate the number 137 correspond to numbers and structures in Pauli's World Clock Dream.

3. The small counting numbers are the archetypes of order; in particular 5 manifesting as Pythagorean geometry and 6 manifesting as Pythagorean arithmetic.

This gives credence to Jung's proposal of an unus mundus or underlying mathematical reality unifying science and mystical experience.

\section{ACKNOWLEDGMENTS}

This research was inspired by Dr. Joseph P. Farrell's webinar on the fine structure constant on his website http://www.gizadeathstar.com. It was in this webinar that I learned about the Flash Forward TV series and novel mentioned in note 3 and some other interesting facts about the fine structure constant. I also wish to acknowledge my friends Maggie Fuller and Sidney Steffens from Galveston, Texas. Maggie told me about Magdalene's Lost Legacy by Margaret Starbird, which was an important reference for this article, and it was Sid's enthusiasm for the number $\pi$ that led me to think about the Lunn-Heisenberg constant. It would be remiss of me not to thank my department head, JoAnn DiGeorgio-Lutz, for giving me a teaching reduction in the Fall semester of 2016 that gave me the time to do this research. Finally, I thank the referees for their references, comments, and suggestions, in response to which I included note 1 on fundamental constants, the paragraph on the g-factor and the anomalous magnetic moment of the electron, the reference to the article by Peres in Physics Today, and Bill Harrington's approximation for $\alpha^{-1}$. 
Philip R. Brown

\section{APPENDIX}

The formula

$$
\tan \frac{\pi}{18}=\frac{1}{\sqrt{3}}=\left(1-4 \sin \frac{\pi}{18}\right)
$$

can be proved using the triple angle identities

$$
\begin{aligned}
\sin (3 \theta) & =3 \sin (\theta)-4 \sin 3(\theta) \\
\cos (3 \theta) & =4 \cos ^{3}(\theta)-3 \cos (\theta) \\
\tan (3 \theta) & =\frac{3 \tan (\theta)-\tan ^{3}(\theta)}{1-3 \tan ^{2}(\theta)}
\end{aligned}
$$

and solving cubic equations; however, the following elegant proof was discovered by the author's student Paxton Martin.

In a $30^{\circ}-60^{\circ}$ right triangle with a hypotenuse of length 2 and sides opposite $30^{\circ}$ and $60^{\circ}$ having lengths 1 and $\sqrt{3}$, respectively, insert a line segment that trisects the $30^{\circ}$ angle and divides the opposite side into lengths labeled $x$ and $y$, as shown in Figure 5.

Figure 5.

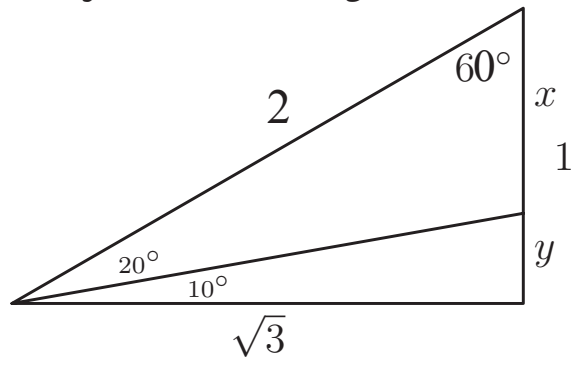

From the sine rule for triangles, it follows that

$$
\frac{x}{\sin \left(20^{\circ}\right)}=\frac{2}{\sin \left(100^{\circ}\right)}
$$

Because $\sin \left(100^{\circ}\right)=\cos \left(10^{\circ}\right)$ and $\sin \left(20^{\circ}\right)=2 \sin \left(10^{\circ}\right) \cos \left(10^{\circ}\right)$ (by an application of the double angle identity for the sine ratio), we find that $x$ $=4 \sin \left(10^{\circ}\right)$. It follows that $y=1-4 \sin \left(10^{\circ}\right)$, and therefore

$$
\tan 10^{\circ}=\frac{1}{\sqrt{3}}\left(1-4 \sin 10^{\circ}\right)
$$

which is formula (36) if the angles are expressed in radian measure. 


\section{NOTES}

1 There are roughly two dozen fundamental physical constants, the majority of which concern the masses of fundamental particles (quarks, leptons, force mediating bosons), and additional constants that also appear as brute facts about Nature that defy deeper explanation including $c$ (the speed of light), $G$ (the universal gravitational constant, $e$ (the charge of the electron), and $\alpha$ (the fine structure constant), which is the topic of this paper.

2 Using cgs units; in SI units this is expressed as

$$
\frac{1}{4 \pi \epsilon_{0}} \frac{e^{2}}{h_{c}}
$$

where $\epsilon_{0}$ is the electric constant or permittivity of free space.

3 For example, in the 2009 TV series Flash Forward (based on the novel of the same title by the Canadian author Robert J. Sawyer in 1999) the entire human population of the planet blacks out for exactly 2 minutes and 17 seconds (a total of 137 seconds) due to a particle accelerator coming online for the first time.

4 If angles are measured in degrees instead of radians, then

$$
\alpha \approx \frac{1}{6^{3}}\left(4 \sin \left(10^{\circ}\right)+5 \tan \left(10^{\circ}\right)\right)
$$

5 This can be obtained using the online base converter (Gang, 1998).

6 The primary polyhedron with pentagonal faces is the regular dodecahedron, which is one of the five Platonic solids. It has twelve pentagonal faces. In Luminet et al. (2003) and Weeks (2004), a geometrical model for the universe as a Poincaré dodecahedral space is presented based on observations of weak wide-angle temperature correlations in the cosmic microwave background. Poincaré dodecahedral space is a non-simply connected 3-manifold formed by "glueing" together the opposite faces of a dodecahedron.

7 The actual values are close to 107.5 and 110.6 , respectively.

8 The Babylonians approximated the ratio of the perimeter of a regular 
hexagon to the circumference of the circumscribed circle by $\frac{24}{25}$. It is not known how they obtained this fraction, which results in $\pi \approx 3.125$ (see Beckman, 1971, pp. 12-13, 21-22).

9 With angles expressed in degrees rather than radians, formula (35) is

$$
\alpha \approx \frac{1}{6^{3}} \frac{\left(1-8 \cos \left(5^{\circ}\right) \sin \left(5^{\circ}\right)\right)\left(1+8 \cos ^{2}\left(5^{\circ}\right)\right)}{\sqrt{3}}
$$

\section{REFERENCES}

Afshordi, N., \& Magueijo, J. (2016, November 18). The critical geometry of a thermal big bang. Physical Review D, 94. https://doi.org/10.1103/PhysRevD.94.101301

Baez, J. (2008). My favorite numbers. http://math.ucr.edu/home/baez/ numbers/

Barrow, J. D. (1999, January). Cosmologies with varying light speed. Physical Review D, 59, 043515. https://doi.org/10.1103/PhysRevD.59.043515

Barton, G. A. (1908). On the Babylonian origin of Plato's nuptial number. Journal of the American Oriental Society, 29, 210-219.

Beckman, P. (1971). A history of pi. Golem Press.

Bogomolny, A. (1996). Golden ratio in regular pentagon. Cut-The-Knot website. https://www.cut-the-knot.org/do_you_know/GoldenRatiolnRegularPentagon. shtml

Bohm, D. (1980). Wholeness and the implicate order. Routledge.

Bohr, N. (1913). On the series spectrum of hydrogen and the structure of the atom. Philosophical Magazine Series 6, 26, 332-335.

Born, M. (1935). The mysterious number 137. Proceedings of the Indian Academy of Sciences A, 2, 533-561.

Brown, P. (2016). Foundations of mathematics: Algebra, geometry, trigonometry and calculus. Mercury Learning and Information.

Campbell, D. T. (1974). Evolutionary epistemology. In P. A. Schilpp (Ed.), The philosophy of Karl Popper (pp. 413-463). Open Court.

Consa, O. (2018). Helical solenoid model of the electron. Progress in Physics, 14(2), $80-89$.

Cowie, L. L., \& Songaila, A. (1995). Astrophysical limits on the evolution of dimensionless physical constants over cosmological time. Astrophysical Journal, 453, 596-598.

Damour, T., \& Dyson, F. (1996). The Oklo bound on the time variation of the finestructure constant revisited. Nuclear Physics B, 480(1), 37-54.

Dattoli, G. (2010). The fine structure constant and numerical alchemy.

Del Castillo-Mussot, M., \& Dias, R. C. (1993). Fuzzy sets and physics. Revista Mexicana de Fisica, 39(2), 295-303.

Eddington, A. S. (1929). The charge of an electron. Proceedings of the Royal Society A, 122(789), 358-369. 
Eddington, A. S. (1931). On the value of the cosmical constant. Proceedings of the Royal Society A, 133(822), 605-615.

Elst, K. (2003). The Koenraad Elst site. http://koenraadelst. bharatvani.org/articles/misc/why108.html

Feynman, R. P. (1988). The strange theory of light and matter. Princeton University Press.

Gang, X. (1998). Base converter. http://wims.unice.fr/wims/en_tool number baseconv.en.html

Gaunt, B. (2000). The bible's awesome number code! Adventures Unlimited Press.

Gellman, J. (2014). Mysticism. Stanford Encyclopedia of Philosophy. http://plato.stanford.edu/entries/ mysticism/

Gilson, J. G. (1996). Calculating the fine-structure constant. Physics Essays, 9(2), 342-353.

Gilson, J. G. (1997). Relativistic wave packing and quantization. Speculations in Science and Technology, 20, 21-31.

Gilson, J. G. (2012). The fine structure constant, a 2oth century mystery.

Gorelik, I. (2009). Fine structure constant collection. OoCities. http://www.oocities.org/igorelik/fine.html

Haas, A. E. (1924). Atomtheorie in elementare darstelling. Walther de Gruyter.

Heisenberg, W. (1934). Bemerkung zur diracshen theorie des positrons. Zitschrift für Physik, 90, 209-231.

Jordan, P. (1937). Die physikalischen weltkonstanten. Die Naturwissenschaften, 25(25), 513-517.

Jung, C. G. (1964). Man and his symbols. Doubleday.

Keppler, J., Aiton, E. J., Duncan, A. M., \& Field, J. V. (1997). The harmony of the world (volume 209). American Philosophical Society.

Kinoshita, T. (1996). The fine structure constant. Reports on Progress in Physics, 59(11), 1459-1492.

Kinoshita, T. (1997). Fine-structure constant obtained from an improved calculation of the electron g-2. IEEE transactions on instrumentation and measurement, 46(2), 108-111.

Koole, W. (1986). The tao of physics. IKON TV in association with BBC TV. https://www.youtube.com/watch?v= kBJFJVZMnlo

Krag, H. (2003). A partial history of the fine structure constant. Archive for History of Exact Sciences, 57, 395-431.

Lanzi, O. (2017). Possible all-pentagon polyhedra. Mathematics Stack Exchange. http://math.stackexchange.com/questions/1609854/possible-all-pentagonpolyhedra

Leeds, M. (2013). Phi, Metatron's cube and the holy number 108. World-Mysteries. http://blog.world-mysteries.com/science/phi-metatrons-cube-and-theholy-number-108/ 
Lewis, G. N., \& Adams, E. Q. (1914). A theory of ultimate rational units; numerical relations between elementary charge, wirkings quantum, constant of stefan's law. Physical Review, 3, 92-102.

Lewis, H. S. (1979). Rosicrucian questions and answers: With complete history of the Rosicrucian Order (volume 1). Supreme Grand Lodge of AMORC.

Luminet, J.-P., Jeffrey R. Weeks, J. R., Riazuelo, A., Lehoucq, R., \& Uzan, J.-P. (2003). Dodecahedral space topology as an explanation for weak wide-angle temperature correlations in the cosmic microwave background. Nature, 425(6958), 593-595.

Lunn, A. C. (1922). Atomic constants and dimensional invariants. Physical Review, 20, 1-14.

Madore, J. (1992). The fuzzy sphere. Classical and Quantum Gravity, 9(1), 69-87.

Michell, J. (2008). The dimensions of paradise: Sacred geometry, ancient science, and the heavenly order on Earth. Simon and Schuster.

Miller, A. I. (2009). 137, Jung, Pauli, and the pursuit of a scientific obsession. W. W. Norton.

Myers, E. G. (2019). High-precision atomic mass measurements for fundamental constants. Atoms, $7(1), 37$.

Olsen, S. (2006). The golden section: Nature's greatest secret. Bloomsbury Publishing,

Onstott, S. (2019). Why 137? Secrets in Plain Sight. http://www.secretsinplainsight.com/ why-137/

Peres, A. (1971). A new pastime-Calculating alpha to one part in a million. Physics Today, 24(11), 9.

Poirier, J. J. (2017). Astrological correspondences in quantum mechanics. Esoteric Science Institute.

https://www.facebook.com/esotericscienceinstitute/posts/1250782138376494:0

Rice, J. (1925). On Eddington's natural unit of the field, and possible relations between it and the universal constants of physics. Philosophical Magazine Series 6, 49(290), 457-463.

RobertLovesPi. (2015). A polyhedron with 36 pentagonal faces. https://robertlovespi.net/2015/12/16/a-polyhedron-with-36-pentagonal-faces

RobertLovesPi. (2016). Three polyhedra with only pentagonal faces. https://robertlovespi.net/2016/08/02/two-polyhedra-with-only-pentagonal-faces

Robertson, R. (1995). Jungian archetypes: Jung, Gödel, and the history of archetypes. Nicolas-Hays.

Savedoff, M. P. (1956). Physical constants in extra-galactic nebulae. Nature, 178, 688-689.

Scholem, G. (1990). Origins of the Kabbalah. Princeton University Press.

Schönfeld, E., \&Wilde, P. (2008). Electron and fine structure constant II. Metrologia, 45(3), 342-355.

Schönfeld, E., \& Wilde, P. (2012). A new theoretical derivation of the fine structure constant. Progress in Physics, 1, 3-5.

Sherbon, M. A. (2008). Constants of nature from the dynamics of time. 
Sherbon, M.A. (2014). Fundamental nature of the fine-structure constant. International Journal of Physical Research, 2(1), 1-9.

Singer, N., Shouldice, A., Ye, L., Daske, T., Dobcsanyi, P., Manna, D., Chan, O.-Y., \& Borwein, J. (1915). Inverse symbolic calculator. University of Newcastle, New South Wales. https://isc.carma.newcastle.edu.au/

Sommerfeld, A. (1915). Die feinstruktur der Wasserstoff und der Wasserstofähnlichen Linien (pp. 459-500). Akademie der Wissenschaften.

Sommerfeld, A. (1916). Zur quantentheorie der spektrallinien. Annalen der Physik, 51, 1-94.

Sperry, R. W. (1987). Structure and significance of the consciousness revolution. The Journal of Mind and Behavior, 8, 7-65.

Stanbury, P. (1983). The alleged ubiquity of $\pi$. Nature, 304(5921), 11.

Starbird, M. (2003). Magdalene's lost legacy: Symbolic numbers and the sacred union in Christianity. Bear \& Co.

Várlaki, P., \& Nádai, L. (2008). Number archetypes and 'background' control theory concerning the fine structure constant. Acta Polytechnica Hungarica, 5(2), 71-104.

Várlaki, P., \& Rudas, I. J. (2009). Twin concept of fine structure constant as the 'self number-archetype' in perspective of the Pauli-Jung correspondence. Acta Polytechnica Hungarica, 6(22), 77-108.

Várlaki, P., Nádai, L., Bokor, J., \& Rövid, A. (2009a). Controlling-observing interpretation of the fine structure constant. In Proceedings of the IEEE 13th International Conference on Intelligent Engineering Systems (pp. 61-71. IEEE Press.

Várlaki, P., Rudas, I. J., \& Fuller, R. (2009b). Fine structure constant-A possibilistic approach. In J. Fodor J. \& J. Kacprzyk (Eds.). (2009b). Towards Intelligent Engineering and Information Technology, 243 of Studies in Computational Intelligence, pages 665-679. Springer.

Webb, J. K., Flambaum, V. V., Churchill, C. W., Drinkwater, M. J., \& Barrow, J. D. (1998). Time variation of the fine structure constant. Physical Review Letters, 82(5), 884-887.

Webb, J. K., Murphy, M. T., Flambaum, V. V., Dzuba, V. A., Churchill, C. W., Barrow, J. D., Prochasta, J. X., \& Wolfe A. M. (2001, August). Further evidence for cosmological evolution of the fine structure constant. Physical Review Letters, 87, 091301.

Webb, J. K., King, J. A., Murphy, M. T., Flambaum, V. V., Carswell, R. F., Bainbridge, M. B., Wilczynska, M. R., \& Koch, F. E. (2012). Spatial variation in the finestructure constant-New results from VLT/UVES. Monthly Notices of the Royal Astronomical Society, 422, 3370-3414.

Weeks, J. (2004). The Poincaré dodecahedral space and the mystery of the missing fluctuations. Notices of the AMS, 51(6), 610-619.

Weisstein, E. W. (1999). Pentagonal hexecontahedron. Wolfram Math-World. http://mathworld.wolfram.com/PentagonalHexecontahedron.html 
Wikipedia. (n.d.). Casting out nines. https://en.wikipedia.org/wiki/Casting|_out|_nines

Wikipedia. (2016). Digital root. https://en.wikipedia.org/wiki/Digital/_root Wikipedia. (2017). 108 (number). https://en.wikipedia.org/wiki/108/_(number) Wikipedia. (2018). Pythagorean triple. https://en.wikipedia.org/wiki/ Pythagorean/_triple Wisnefsky, M. Y. (1993). 72 'Names' of G-d. http://www.chabad.org/kabbalah/ article_cdo/aid/1388270/jewish/72-Names-of-G-d.htm 